\title{
Role of hydroxychloroquine in multidrug treatment of COVID-19
}

\author{
Peter A. McCullough ${ }^{1, *}$, Raphael B. Stricker ${ }^{2}$, Harvey A. Risch ${ }^{3}$ \\ ${ }^{1}$ Truth for Health Foundation, Tucson, AZ, USA \\ ${ }^{2}$ Union Square Medical Associates, San Francisco, CA, USA \\ ${ }^{3}$ Yale School of Public Health, New Haven, CT 06510, USA \\ *Correspondence: Peteramccullough@gmail.com (Peter A. McCullough)
}

\section{DOI:10.31083/j.rcm2203063}

This is an open access article under the CC BY 4.0 license (https://creativecommons.org/licenses/by/4.0/).

Submitted: 16 September 2021 Accepted: 17 September 2021 Published: 24 September 2021

\section{Keywords}

SARS-CoV-2; COVID-19; Hydroxychloroquine; Ambulatory treatment; Mortality

Hydroxychloroquine is the most widely prescribed intracellular anti-infective for human SARS-CoV-2 infection and COVID-19 syndrome. There have been 296 studies, 220 of which are peer reviewed, 246 comparing treatment and control groups [1]. This agent is successfully used in both prophylaxis and early therapy (Fig. 1). As a general principle, the earlier hydroxychloroquine is started in the course of illness, the larger treatment effects can be observed. These effects are greatly enhanced by the use of agents in combination to address SARS-CoV-2 replication, cytokine storm, and thrombosis [2, 3]. Early treatment of SARS-CoV-2 infection has the largest opportunity to control the outbreak since efforts are placed on those acutely ill with COVID-19 where there are interventions to control spread of disease, reduce the intensity and duration of illness, generate natural immunity, and prevent hospitalization and death, resulting in greater population protection from SARS-CoV-2 [4].

In this issue of Reviews in Cardiovascular Medicine, a paper from Million et al. [5] describes the impact of hydroxychloroquine as a direct treatment for early COVID-19 in ambulatory patients presenting to an expert day hospital (Institut Hospitalo-Universitaire Mediterraneé Infection) for early therapy with the goal of reducing hospitalization and death. Among 10,429 patients who took on average 4 days to present for medical care and another day to receive SARS-CoV-2 test results, 8315 were treated with hydroxychloroquine and azithromycin and this group had an observed case fatality fraction of $0.06 \%$. The combination of hydroxychloroquine and azithromycin was associated with a 93\% reduction in mortality (adjusted $\mathrm{OR}=0.17,95 \% \mathrm{CI}$ $0.06-0.48, p=0.0007, \mathrm{n}=2015$ age $\geq 60$ years). There were 5 deaths among the 8315 patients who received hydroxychloroquine and azithromycin and 11 among the 2114 who

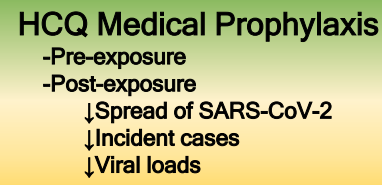

HCQ-Based Multidrug Treatment Regimens $\downarrow$ Spread of SARS-CoV2 by enabling full home quarantine $\downarrow$ Intensity and duration of symptoms

Risk of hospitalization

$\downarrow$ Risk of death

$\uparrow$ Population natural immunity

-Robust

-Complete

-Durable

$\mathrm{HCQ}=$ hydroxychloroquine

Fig. 1. Roles of hydroxychloroquine in the prevention and treatment of SARS-CoV-2 infection and the COVID-19 syndrome.

received other treatments $(p<0.0001)$. These data are consistent with four large studies presented by the authors in a meta-analysis of 32,124 patients from five countries, showing that early treatment with hydroxychloroquine was associated with a $69 \%$ reduction in mortality [5]. These data are also consistent with U.S. primary care cohorts that have demonstrated $\sim 85 \%$ reductions in both hospitalization and death with various multidrug regimens, most with hydroxychloroquine as a base [6].

The large study conducted over nine months from Million and colleagues demonstrates that very low rates of mortality can be achieved in a dedicated outpatient "hospital" even if therapy is started relatively late into the course of viral replication [7]. A weakness of the study by Million et al. is that it is from a single center and not randomized. These findings are complemented by the contemporary early use of hydroxychloroquine in combination with anti-inflammatory agents, and antithrombotics in some cases to extend the range of hydroxychloroquine to higher risk patients and reduce the risks of both hospitalization and death [7]. Thus in aggregate, the 
body of literature supporting early ambulatory therapy for high-risk COVID-19 patients is compelling and at this point, only large ( $n>20,000)$ randomized multidrug randomized trials could better inform the community on the most ideal drug combination for future early ambulatory therapy [7]. At the time of this writing, the World Health Organization, Centers for Disease Control, Infectious Disease Society of America, and European Society of Clinical Microbiology and Infectious Diseases have not comprehensively addressed early ambulatory therapy for patients with COVID-19 and have made no recommendations for any single agent or combination regimens. Hence the present efforts on defining the roles of single and multiple drugs are critical in the pandemic response.

\section{Author contributions}

PAM drafted, edited, finalized the manuscript, RBS provided edits and references, HAR provided edits

\section{Ethics approval and consent to participate Not applicable.}

\section{Acknowledgment}

Not applicable.

\section{Funding}

This research received no external funding.

\section{Conflict of interest}

The authors declare no conflict of interest.

\section{References}

[1] Anonymous. HCQ for COVID-19: real-time meta analysis of 265 studies. Covid Analysis, Jul 15, 2021, Version 126. Avaliable at: https://hcqmeta.com/hcq-meta.pdf (Accessed: 17 July 2021).

[2] McCullough PA, Kelly RJ, Ruocco G, Lerma E, Tumlin J, Wheelan $\mathrm{KR}$, et al. Pathophysiological Basis and Rationale for Early Outpatient Treatment of SARS-CoV-2 (COVID-19) Infection. The American Journal of Medicine. 2021; 134: 16-22.

[3] McCullough PA, Alexander PE, Armstrong R, Arvinte C, Bain AF, Bartlett RP, et al. Multifaceted highly targeted sequential multidrug treatment of early ambulatory high-risk SARS-CoV-2 infection (COVID-19). Reviews in Cardiovascular Medicine. 2020; 21: $517-530$.

[4] McCullough PA, Vijay K. SARS-CoV-2 infection and the COVID19 pandemic: a call to action for therapy and interventions to resolve the crisis of hospitalization, death, and handle the aftermath. Reviews in Cardiovascular Medicine. 2021; 22: 9-10.

[5] Million M, Lagier J, Tissot-Dupont H, Ravaux I, Dhiver C, Tomei C . Early Combination Therapy with Hydroxychloroquine and Azithromycin Reduces Mortality in 10,429 COVID-19 Outpatients. Reviews in Cardiovascular Medicine. 2021. (in press)

[6] Procter BC, Ross C, Pickard V, Smith E, Hanson C, McCullough PA. Early Ambulatory Multidrug Therapy Reduces Hospitalization and Death in High-Risk Patients with SARS-CoV-2 (COVID19). International Journal of Innovative Research in Medical Science. 2021; 6: 219-221

[7] McCullough PA. The Reply. The American Journal of Medicine. 2021; 134: e343-e344. 